\title{
Properties of the Scalar Universal Equations
}

\author{
J.A. Mulvey \\ Department of Mathematical Sciences \\ University of Durham, \\ South Road, \\ Durham, DH1 3LE, UK \\ email: J.A.Mulvey@durham.ac.uk
}

19th September 1995

\section{Preprint DTP $/ 95 / 53$}

\begin{abstract}
The variational properties of the scalar so-called "Universal" equations are reviewed and generalised. In particular, we note that contrary to earlier claims, each member of the Euler hierarchy may have an explicit field dependence. The Euler hierarchy itself is given a new interpretation in terms of the formal complex of variational calculus, and is shown to be related to the algebra of distinguished symmetries of the first source form.
\end{abstract}




\section{Introduction}

The Universal Field models proposed in [1, 2, 3, 国 are a class of integrable field theories with a wide variety of attractive features:

- they may be formulated in an arbitrary number of space-time dimensions;

- they are either diffeomorphism or reparametrisation invariant in the dependent variables;

- they are Lorentz and Euclidean invariant (and in fact $G L(m, \mathbb{R})$ invariant) in the independent variables;

- they are derived from an infinite number of inequivalent Lagrangians.

Furthermore, the scalar versions of the theory have been shown to be linearisable. One of the scalar theories is a direct generalisation of the Bateman equation [5] and is linearisable by a Legendre transformation in the manner described in $\mid$ |

The business at hand is to describe and explain the strange variational properties of the scalar Universal equations. Consider a scalar field $\phi$ dependent on $m$ space(-time) coordinates. The variational derivation of the Universal equations presented in [3] is based on the idea of the generic Euler hierarchy. Consider a Lagrangian density $\mathcal{L}_{0}$ which only depends on the first derivatives of $\phi$. Compute the variation of this and construct a new function,

$$
\mathcal{L}_{1}=F_{1} \mathcal{E} \mathcal{L}_{0},
$$

where $\mathcal{E}$ is the Euler variation and $F_{1}$ is some real-valued function dependent only on the first derivatives of $\phi$. Then consider $\mathcal{L}_{1}$ to be the Lagrangian for a new equation, $\mathcal{E} \mathcal{L}_{1}$. Continue the process to arrive at:

$$
\mathcal{L}_{k}=F_{k} \mathcal{E} \mathcal{L}_{k-1} .
$$

This sequence terminates at the $(m+1)$ th stage: $\mathcal{E} \mathcal{L}_{m}=0$. Furthermore, at the $m$ th stage the resulting Euler-Lagrange expression factorises, and one of the factors is "universal" - 
independent of the details of the initial and intermediate Lagrangians. On setting this EulerLagrange form to zero, we arrive at an equation of motion equivalent to the Monge-Ampère expression,

$$
\Delta_{M-A}=\operatorname{det} H=0,
$$

where $H$ is the Hessian matrix of the dependent variable $\phi: H_{i j}=\phi_{i j}$.

The most interesting special cases of this construction are referred to in [3] as generalised Bateman equations. The idea is to use an initial Lagrangian $\mathcal{L}_{0}$ which is homogeneous of degree one in the first derivatives of $\phi$, and to restrict all the $F_{k}$ to have this property too. Performing the procedure described above leads to the cessation of the hierarchy a stage earlier than described above: $\mathcal{E L}_{m-1}=0$. Remarkably, the penultimate Euler-Lagrange expression $\mathcal{E} \mathcal{L}_{m-2}$ is again a product, and again one of the its factors is "universal". Setting this expression to zero gives the Universal Bateman Equation of [1] and sequels.

The equation can be written,

$$
\Delta=\operatorname{det}\left(\begin{array}{clll}
0 & \phi_{x_{1}} & \ldots & \phi_{x_{m}} \\
\phi_{x_{1}} & \phi_{x_{1} x_{1}} & \ldots & \phi_{x_{1} x_{m}} \\
\vdots & \vdots & \ddots & \vdots \\
\phi_{x_{m}} & \phi_{x_{m} x_{1}} & \ldots & \phi_{x_{m} x_{m}}
\end{array}\right)=0
$$

or in components,

$$
\Delta=\varepsilon_{i_{1} \ldots i_{m}} \varepsilon_{j_{1} \ldots j_{m}} \phi_{i_{1}} \phi_{j_{1}} \phi_{i_{2} j_{2}} \ldots \phi_{i_{m} j_{m}}=0 .
$$

The component form makes it easy to verify that $\Delta$ can also be expressed as:

$$
\Delta=\operatorname{tr}\left(G H^{\dagger}\right)
$$

where the matrix $G$ has components

$$
G_{i j}=\phi_{i} \phi_{j}
$$

and

$$
H^{\dagger}=\operatorname{adj}(H)
$$


is the classical adjoint matrix of $H$. Alternatively, we could define a new matrix $U$ such that (1.6) is equivalent to the equation:

$$
\Delta=\operatorname{tr}(U H) .
$$

The explicit form of $U$ is easily deduced from (1.5). It is:

$$
U=\varepsilon_{i_{1} \ldots i_{m}} \varepsilon_{j_{1} \ldots j_{m}} \phi_{i_{1}} \phi_{j_{1}} \phi_{i_{2} j_{2}} \ldots \phi_{i_{m-1} j_{m-1}} .
$$

A supplementary assumption is that $\operatorname{det} H$ is non-vanishing.

Our mission is to try to understand and generalise the generic and Bateman hierarchies described above from the point of view of the standard theory of variational calculus as presented in, for example, the book by Olver [6]. In particular, we wish to know under what circumstances we can introduce dependence on the field $\phi$ into the initial Lagrangian and the multiplier functions $F_{k}$. We will find that only in certain circumstances does the Euler hierarchy define a "universal" theory. In addition, we will think about what the iterative procedure means in terms of the formal variational complex, described in [6] or the work of Anderson [7]. We will find that the iterated equations of motion are related to determining equations for distinguished symmetries of higher members of the hierarchy, and we will justify this connection with a calculation of the generalised symmetries of the Universal equations.

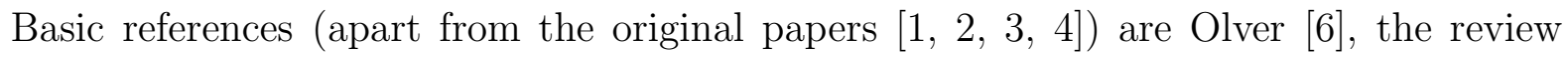
articles by Anderson [0, 8] and his recent work with Pohjanpelto [9, 10]. Another view of the universal equations and their variational symmetry properties is given by Grigore [11, 12].

\section{Lagrangian Properties}

We begin by analysing the Euler hierarchy in the language of variational calculus presented in [6, [7]. Initially, we will only assume that the initial Lagrangian depends on the field and its first derivatives. In the language of jet bundles [13], we ask that the Lagrangian is a smooth function $\mathcal{L}_{0}: J^{1} \pi \rightarrow \mathbb{R}$, where $\mathrm{J}^{1} \pi$ is the first jet bundle of the bundle $\pi$. In this case we are restricting our attention to the trivial bundle $\pi: \mathbb{R}^{m+1} \rightarrow \mathbb{R}^{m}$. We make no assumptions about any symmetries of the resulting action. This language will be useful later. 
From $\mathcal{L}_{0}$, we derive an Euler-Lagrange form:

$$
\Delta_{1}=\mathrm{D}_{\mathcal{L}_{0}}^{*}(1)=\mathcal{E} \mathcal{L}_{0} .
$$

From this we construct the next Lagrangian,

$$
\mathcal{L}_{1}=F_{1} \Delta_{1}
$$

where we assume that $F_{1}$ depends only on $\phi$ and its first derivatives. The next source form follows readily using the product formula:

$$
\mathcal{E}\left(\mathcal{F}_{1} \mathcal{F}_{2}\right)=\mathrm{D}_{\mathcal{F}_{1}}^{*}\left(\mathcal{F}_{2}\right)+\mathrm{D}_{\mathcal{F}_{2}}^{*}\left(\mathcal{F}_{1}\right),
$$

where $D^{*}$ indicates the adjoint of the Fréchet derivative [6]. In this case:

$$
\Delta_{2}=\mathcal{E} \mathcal{L}_{1}=\mathrm{D}_{\Delta_{1}}^{*}\left(F_{1}\right)+\mathrm{D}_{F_{1}}^{*}\left(\Delta_{1}\right) .
$$

Now, the Helmholtz condition of the calculus of variations states that an equation is an exact Euler-Lagrange form if and only if its Fréchet derivative is self-adjoint. Hence:

$$
\begin{aligned}
\mathrm{D}_{\Delta_{1}}^{*}\left(F_{1}\right) & =\mathrm{D}_{\Delta_{1}}\left(F_{1}\right) \\
& =\frac{\partial \Delta_{1}}{\partial \phi_{J}} D_{J}\left(F_{1}\right)
\end{aligned}
$$

The second term on the right-hand side of (2.4) just turns out to be,

$$
\mathrm{D}_{F_{1}}^{*}\left(\Delta_{1}\right)=\Delta_{1} \frac{\partial F_{1}}{\partial \phi}-D_{i}\left(\frac{\partial F_{1}}{\partial \phi_{i}} \Delta_{1}\right),
$$

since $F_{1}$ only depends on $\phi$ and its first derivatives.

Putting (2.5) and (2.6) into (2.4), a short calculation tells us that:

$$
\begin{aligned}
\Delta_{2}= & \Delta_{1} \mathcal{E} F_{1}+\frac{\partial \Delta_{1}}{\partial \phi}\left(F_{1}-\frac{\partial F_{1}}{\partial \phi_{i}} \phi_{i}\right)+\frac{\partial \Delta_{1}}{\partial \phi_{i}} \frac{\partial F_{1}}{\partial \phi} \phi_{i} \\
& +\frac{\partial \Delta_{1}}{\partial \phi_{i j}}\left(D_{i}\left(\frac{\partial F_{1}}{\partial \phi} \phi_{j}\right)+D_{i}\left(\frac{\partial F_{1}}{\partial \phi_{k}}\right) \phi_{j k}\right) .
\end{aligned}
$$

The important point to notice is that this construction guarantees that there are no derivatives of order higher than two in the resulting equation of motion. This means that when 
the process is continued, the analysis is essentially unchanged. We are led to the iterative formula for the $(k+1)$ th Euler-Lagrange form:

$$
\begin{aligned}
\Delta_{k+1}= & \Delta_{k} \mathcal{E} F_{k}+\frac{\partial \Delta_{k}}{\partial \phi}\left(F_{k}-\frac{\partial F_{k}}{\partial \phi_{i}} \phi_{i}\right)+\frac{\partial \Delta_{k}}{\partial \phi_{i}} \frac{\partial F_{k}}{\partial \phi} \phi_{i} \\
& +\frac{\partial \Delta_{k}}{\partial \phi_{i j}}\left(D_{i}\left(\frac{\partial F_{k}}{\partial \phi} \phi_{j}\right)+D_{i}\left(\frac{\partial F_{k}}{\partial \phi_{l}}\right) \phi_{j l}\right) .
\end{aligned}
$$

There is no such recursive definition if $F_{k}$ depends on second or higher derivatives.

We are interested in sequences generated by this kind of recursion which terminate after a finite number of iterations. The expression (2.8) simplifies greatly if we restrict attention to $F_{k}$ that are (1) independent of $\phi$, and (2) homogeneous of degree one in the first derivatives. Then we find:

$$
\Delta_{k+1}=\Delta_{k} \mathcal{E} F_{k}+\frac{\partial \Delta_{k}}{\partial \phi_{i j}} D_{i}\left(\frac{\partial F_{k}}{\partial \phi_{l}}\right) \phi_{j l}
$$

or more symmetrically,

$$
\Delta_{k+1}=\left(\frac{\partial \Delta_{k}}{\partial \phi_{k l}} \phi_{i k} \phi_{j l}-\Delta_{k} \phi_{i j}\right) \frac{\partial^{2} F_{k}}{\partial \phi_{i} \partial \phi_{j}} .
$$

This is precisely the recurrence found by Fairlie and Govaerts ([3]) in their treatment of the generic hierarchy. They found that if $\mathcal{L}_{0}$ and all the $F_{k}$ were independent of $\phi$ then the sequence terminated in a Monge-Ampère equation. This sequence only produces such a result if it is assumed that the initial Lagrangian is independent of $\phi$, in which case the condition (2) can be relaxed anyway, so there is no new information. Otherwise, (2.9) fails to reproduce the generic hierarchy. Termination of the hierarchy seems to depend on certain linear-algebraic properties induced by the fact that the second derivatives appear homogeneously at each stage of the sequence [3]. This does not happen if the $F_{k}$ depend on $\phi$. So far, all attempts to generalise the construction of the generic hierarchy by relaxing this requirement have failed. Sample computer calculations carried out on MAPLE show that such constructions do not terminate in universal, exact source forms at either the $(m-1)$ th or $m$ th stages.

We proceed to generate the equations of the generic hierarchy under the restriction that the $\mathcal{L}_{0}$ and $F_{k}$ are $\phi$-independent. We know that the first equation (2.1) has the explicit 
form,

$$
\Delta_{1}=-\frac{\partial^{2} \mathcal{L}_{0}}{\partial \phi_{i} \partial \phi_{j}} \phi_{i j}
$$

which can be written:

$$
\Delta_{1}=-\operatorname{tr}\left(H M_{0}\right),
$$

where $M_{0}$ is the Hessian matrix of $\mathcal{L}_{0}$ with respect to its dependence on first derivatives of $\phi$. It is then a straightforward matter to apply the recursion relation (2.10) to this starting term. Using the notation,

$$
\begin{aligned}
{\left[M_{k}\right]_{i j} } & =\frac{\partial^{2} F_{k}}{\partial \phi_{i} \partial \phi_{j}}, \\
P_{k} & =H M_{k},
\end{aligned}
$$

the first few terms of the hierarchy are:

$$
\begin{aligned}
\Delta_{1} & =-\operatorname{tr}\left(P_{0}\right), \\
\Delta_{2} & =\operatorname{tr}\left(P_{0}\right) \operatorname{tr}\left(P_{1}\right)-\operatorname{tr}\left(P_{0} P_{1}\right), \\
\Delta_{3} & =-\operatorname{tr}\left(P_{2}\left(P_{0} P_{1}+P_{1} P_{0}\right)\right)+\operatorname{tr}\left(P_{0}\right) \operatorname{tr}\left(P_{1} P_{2}\right)+\operatorname{tr}\left(P_{1}\right) \operatorname{tr}\left(P_{0} P_{2}\right) \\
& +\operatorname{tr}\left(P_{2}\right) \operatorname{tr}\left(P_{0} P_{1}\right)-\operatorname{tr}\left(P_{0}\right) \operatorname{tr}\left(P_{1}\right) \operatorname{tr}\left(P_{2}\right), \\
\Delta_{4} & =-\operatorname{tr}\left(P_{3}\left(P_{1} P_{2} P_{0}+P_{0} P_{1} P_{2}+P_{2} P_{0} P_{1}+P_{0} P_{2} P_{1}+P_{1} P_{0} P_{2}+P_{2} P_{1} P_{0}\right)\right) \\
& +\operatorname{tr}\left(P_{1} P_{2}\right) \operatorname{tr}\left(P_{0} P_{3}\right)+\operatorname{tr}\left(P_{2} P_{3}\right) \operatorname{tr}\left(P_{0} P_{1}\right)+\operatorname{tr}\left(P_{1} P_{3}\right) \operatorname{tr}\left(P_{0} P_{2}\right) \\
& +\operatorname{tr}\left(P_{0}\right)\left(\operatorname{tr}\left(P_{1} P_{2} P_{3}\right)+\operatorname{tr}\left(P_{3} P_{2} P_{1}\right)\right)+\operatorname{tr}\left(P_{1}\right)\left(\operatorname{tr}\left(P_{0} P_{2} P_{3}\right)+\operatorname{tr}\left(P_{3} P_{2} P_{0}\right)\right) \\
& +\operatorname{tr}\left(P_{2}\right)\left(\operatorname{tr}\left(P_{0} P_{1} P_{3}\right)+\operatorname{tr}\left(P_{3} P_{1} P_{0}\right)\right) \\
& -\operatorname{tr}\left(P_{0}\right) \operatorname{tr}\left(P_{1}\right) \operatorname{tr}\left(P_{2} P_{3}\right)-\operatorname{tr}\left(P_{0}\right) \operatorname{tr}\left(P_{2}\right) \operatorname{tr}\left(P_{1} P_{3}\right)-\operatorname{tr}\left(P_{1}\right) \operatorname{tr}\left(P_{2}\right) \operatorname{tr}\left(P_{0} P_{3}\right) \\
& -\operatorname{tr}\left(P_{3}\right) \Delta_{3}
\end{aligned}
$$

The subsequent members of the hierarchy become combinatorially more complicated.

We can use the recursion relation as the basis for a plausibility argument that demonstrates the vanishing of the $\Delta_{k}$ at a certain stage, and hence when the $F_{k}$ is the characteristic 
of a conservation law. We wish to know when $\Delta_{k}$ vanishes for any possible choices of $F_{k}$. (Of course, we are only interested in those cases when the Hessian of $F_{k}$ is non-vanishing.) This amounts to solving the matrix differential equation,

$$
H \Gamma^{k} H=\Delta_{k} H
$$

where,

$$
\Gamma_{i j}^{k}=\frac{\partial \Delta_{k}}{\partial \phi_{i j}} .
$$

The equation (2.15) is solved by any $\Delta_{k}$ of the form $\Delta=$ (some factor) $\times \operatorname{det} H$, where "some factor" is independent of the second derivatives of $\phi$. We know from the variational calculation that the second derivatives only enter the source forms such that $\Delta_{k}$ is homogeneous of degree $k$ in the second derivatives. Therefore, this solution can only work at the $m$ th stage. We will see in the next section that any function of the first derivatives of $\phi$ is the characteristic of a generalised symmetry of $\Delta_{M-A}$. It follows immediately that taking an Euler variation of $\Delta_{M-A}$ as described above will give a zero result.

A more rigorous proof of these results is presented in [3] using an explicit calculation.

This completes the discussion of the generic case. Now we turn to the Bateman case. The reader should keep in mind Euler's theorem on homogeneous functions, which states that, if $\mathcal{F}(\mathbf{x})$ is homogeneous of degree $\alpha$ in its arguments $\mathbf{x}=\left(x_{1}, \ldots, x_{n}\right)$, then:

$$
\sum_{i=1}^{n} x_{i} \frac{\partial \mathcal{F}}{\partial x_{i}}=\alpha \mathcal{F}
$$

In our case, we assume that $\mathcal{L}_{0}$ is homogeneous of degree one in the first derivatives of $\phi$, meaning that,

$$
\phi_{i} \frac{\partial \mathcal{L}_{0}}{\partial \phi_{i}}=\mathcal{L}_{0} .
$$

It is easy to derive further homogeneity properties of the Euler variation and the derivatives of $\mathcal{L}_{0}$.

In particular, the first derivative of $\mathcal{L}_{0}$ with respect to $\phi_{i}$ is homogeneous of degree zero:

$$
\phi_{i} \frac{\partial^{2} \mathcal{L}_{0}}{\partial \phi_{i} \partial \phi_{j}}=0,
$$


which implies the singularity condition:

$$
\operatorname{det}\left(M_{0}\right)=0
$$

since $\phi$ is arbitrary.

So we proceed to discussion of the Bateman hierarchy. Now we will assume that the initial Lagrangian and the $F_{k}$ have arbitrary dependence on $\phi$ and are homogeneous of degree one in first derivatives of $\phi$. This greatly civilises the shapes of both $\Delta_{1}$ and $\Delta_{2}$ from their original forms (2.1) and (2.7). If we use the various properties stemming from Euler's theorem, we recalculate $\Delta_{1}$ and $\Delta_{2}$ to be,

$$
\begin{aligned}
& \Delta_{1}=-\frac{\partial^{2} \mathcal{L}_{0}}{\partial \phi_{i} \phi_{j}} \phi_{i j} \\
& \Delta_{2}=\left(\frac{\partial \Delta_{1}}{\partial \phi_{k l}} \phi_{i k} \phi_{j l}-\Delta_{1} \phi_{i j}\right) \frac{\partial^{2} F_{1}}{\partial \phi_{i} \partial \phi_{j}}
\end{aligned}
$$

from which we deduce precisely the same recurrence (2.10) without the restriction that the $F_{k}$ need be independent of $\phi$.

The recursive procedure now defines a set of equations identical in form to (2.14), and so

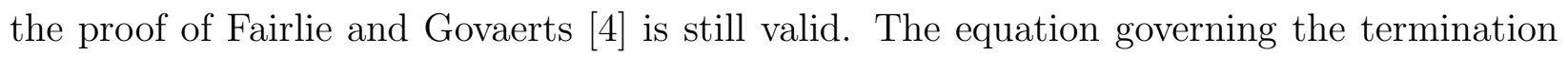
of the sequence (2.15) is satisfied by (1.5).

There is a nice interpretation of all this in terms of the theory of the Euler-Lagrange complex studied in [7]. Let $\Omega^{*}$ denote the algebra of differential forms on the infinite jet bundle $\mathrm{J}^{\infty} \pi$. This algebra is bigraded into,

$$
\Omega^{*}=\bigoplus_{r, s} \Omega^{(r, s)}
$$

where the spaces $\Omega^{(r, s)}$ contain forms with the local coordinate expression:

$$
f[\phi] d x^{i_{1}} \wedge \ldots \wedge d x^{i_{r}} \wedge \theta_{J_{1}}^{\alpha_{1}} \wedge \ldots \wedge \theta_{J_{s}}^{\alpha_{s}}
$$

the forms $\theta$ being the various contact one-forms. The various differentials and subcomplexes of this space are discussed in [7]. Alternatively, see the book by Saunders [13]. 
The starting Lagrangian is a form $\lambda_{0} \in \Omega^{(m, 0)}$. The corresponding variation is the source form $\Delta_{1} d \phi \wedge \omega=\mathcal{E}(\lambda) \in \Omega^{m, 1}$. Now consider taking a Lie derivative (denoted by $\mathrm{L}$ ) of $\Delta_{1}$ with respect to the prolongation of some vector field $X_{1}$ whose evolutionary form is $X_{F_{1}}$, with characteristic $F_{1}$. For an arbitrary equation $\Delta$, we have the Cartan formula:

$$
\left.\left.\mathrm{L}_{\mathrm{pr} X_{1}}(\Delta d \phi \wedge \omega)=\delta_{V}\left(X_{F_{1}}\right\lrcorner \Delta d \phi \wedge \omega\right)+X_{F_{1}}\right\lrcorner \delta_{V}(\Delta d \phi \wedge \omega) .
$$

The differentials $\delta_{V}$ are those of the Euler-Lagrange complex defined in [6] or [7]. A proof of (2.23) can be found in [9]. Each term has a simple interpretation in the calculus of variations. The left hand side vanishes identically if $X_{1}$ is a distinguished symmetry of $\Delta$. The first term on the right is an Euler variation, and so it vanishes if $F_{1}$ is the characteristic of a conservation law. Finally, the $\delta_{V}$ in the third term is just a Helmholtz operator, so it vanishes if $\Delta$ is an exact Euler-Lagrange form. Not surprisingly, this is the central formula in the recent studies of the generalised Noether theorem by Anderson and Pohjanpelto [9, 10].

Returning to our example, (2.23) takes on a particularly simple form when applied to $\Delta_{1}$. Since $\Delta_{1}$ is an exact Euler-Lagrange form, the Helmholtz term vanishes and we are left with the form $\Delta_{2}$ as defined in (2.4). This interpretation holds for all $\Delta_{k}$, so we can view the Euler hierarchy as repeated application of the Lie derivative to successive source forms. So the recursive definition becomes:

$$
\Delta_{k+1} d \phi \wedge \omega=\mathrm{L}_{\operatorname{prv}_{F_{k}}}\left(\Delta_{k} d \phi \wedge \omega\right)
$$

The "universal" theory rests on the observation that at a certain stage the all source forms so defined, in either the generic or Bateman hierarchy, are equivalent to one another and their flows defined by the Lie derivative are identically zero. In such a situation, the iteration vanishes identically, and this yields a product expansion of the type (2.3) which is equal to zero. This indicates that $\mathcal{L}_{k}$ is a total divergence, and hence $F_{k}$ must be the characteristic of a conservation law. Explicitly, we deduce that any function of the first derivatives of $\phi$ is a generalised symmetry of the Monge-Ampère equation and that any function of homogeneous of weight one in the $\phi_{i}$ but with arbitrary dependence on $\phi$ is a generalised symmetry of the 
Bateman-type Universal equation (1.4). These results will be proved explicitly in the next section.

Changing emphasis, we can view the $F_{k}$ as unknown functions to be determined, and then we can interpret $\Delta_{2}$ as the equation determining the distinguished symmetry algebra of the Euler-Lagrange form $\Delta_{1}$. The third and subsequent source forms $\Delta_{3}, \ldots, \Delta_{m}$ are a set of nested equations determining distinguished symmetry algebras for their immediate predecessors. It would be interesting to know what information, if any, can be gleaned from these higher equations about the symmetries of the original equation $\Delta_{1}$.

\section{Generalised Symmetries}

In order to confirm our understanding of how the generic and Bateman hierarchies terminate, it will be helpful to know more about the generalised symmetries of the equations (1.3) and (1.4). To that end we will carry out a detailed analysis of each of each equation in the manner described in Olver's book [6]. We will look for first-order generalised symmetries - in other words symmetries whose evolutionary characteristic depends on the $x_{i}, \phi$ and the first derivatives of $\phi$. The reader is reminded of the standard expansions of the total derivatives $D_{i}$ and $D_{i j}$ :

$$
\begin{aligned}
D_{i} F & =\frac{\partial F}{\partial x_{i}}+\frac{\partial F}{\partial \phi_{J}} \phi_{J i} \\
D_{i j} F & =\frac{\partial^{2} F}{\partial x_{i} \partial x_{j}}+\frac{\partial^{2} F}{\partial x_{i} \partial \phi_{K}} \phi_{K j}+\frac{\partial F}{\partial \phi_{J}} \phi_{J i j}+\frac{\partial^{2} F}{\partial x_{j} \partial \phi_{J}} \phi_{J i}+\frac{\partial^{2} F}{\partial \phi_{J} \partial \phi_{K}} \phi_{J i} \phi_{K j}
\end{aligned}
$$

where the multi-indices $J, K$ will actually only have length zero or one in the cases we are considering.

We will begin with the equation (1.3). It turns out that the first-order generalised symmetries of this equation span a rather large infinity of possibilities. Using the standard procedure, we seek a generalised symmetry in the evolutionary form,

$$
\mathbf{v}_{Q}=Q \frac{\partial}{\partial \phi}
$$


The symmetry equation is quite straightforward:

$$
\operatorname{prv}_{Q}\left(\Delta_{M-A}\right)=\frac{\partial \Delta_{M-A}}{\partial \phi_{i j}} D_{i j} Q[\phi]=H_{i j}^{\dagger} D_{i j} Q[\phi] .
$$

The symbol pr denotes the infinite prolongation. On expanding the total derivative, we find that the coefficients of the third derivatives of $\phi$ vanish on solutions of (1.3) (after taking into account the first prolongation of the equation of motion) and the first symmetry constraint comes from the term of order $(m+1)$ in the second derivatives, which gives:

$$
H_{i j}^{\dagger} \frac{\partial^{2} Q}{\partial \phi_{k} \partial \phi_{l}} \phi_{i k} \phi_{j l}=0
$$

on solutions. Writing,

$$
\left[H_{Q}\right]_{k l}=\frac{\partial^{2} Q}{\partial \phi_{k} \partial \phi_{l}}
$$

we find that (3.4) becomes:

$$
\operatorname{tr}\left(H^{\dagger} H H_{Q} H\right)=\operatorname{det} H \operatorname{tr}\left(H_{Q} H\right)=0,
$$

and this is identically true on solutions of $\Delta_{M-A}=0$. This pattern continues for the terms of order $m$ in second derivatives, and we find that a $Q$ with arbitrary dependence on the first derivatives is a generalised symmetry for (1.3). This is the expected result given the interpretation of the generic hierarchy and confirms its termination at the $(m+1)$ th stage.

Now we turn our attention to the symmetry algebra of the Universal equation (1.4), which turns out to be a little more restrictive than in the generic case. The determining equation is,

$$
\frac{\partial \Delta}{\partial \phi_{i}}\left(D_{i} Q[\phi]\right)+\frac{\partial \Delta}{\partial \phi_{(i j)}}\left(D_{i j} Q[\phi]\right)=0
$$

on solutions of $\Delta=0$. Again, we will only look for first-order generalised symmetries. This should be sufficient to confirm our interpretation of the Bateman hierarchy in the previous section. Furthermore, experience with the two dimensional case [5] suggests that the symmetries dependent on first derivatives are responsible for the linearisability property.

Given this assumption, (3.7) expands to become,

$$
\varepsilon_{i_{1} \ldots i_{m}} \varepsilon_{j_{1} \ldots j_{m}} \phi_{i_{1}} \phi_{j_{1}} \phi_{i_{2} j_{2}} \ldots \phi_{i_{m} j_{m}}+(m-1) U_{k l} \delta_{(i j)(k l)} D_{i j} Q=0,
$$


where,

$$
U_{k l}=\varepsilon_{i_{1} \ldots i_{m-1} k} \varepsilon_{j_{1} \ldots j_{m-1} l} \phi_{i_{1}} \phi_{j_{1}} \phi_{i_{2} j_{2}} \ldots \phi_{i_{m-1} j_{m-1}}
$$

is the sort of matrix appearing in (1.9).

Following the usual algorithm, we must set the left side of (3.8) to zero order-by-order in the derivatives, taking into account the equation $\Delta=0$. Using the first prolongation of $\Delta$, we find that third order derivatives automatically vanish, and so the first task is to find the condition for the vanishing of terms of order $m$ in the second derivatives of $\phi$.

Extracting the relevant term from (3.8), we define a matrix $S$ such that:

$$
\begin{aligned}
S_{k l} \frac{\partial^{2} Q}{\partial \phi_{k} \partial \phi_{l}} & =(m-1) H_{k i} U_{i j} H_{j l} \frac{\partial^{2} Q}{\partial \phi_{k} \partial \phi_{l}} \\
& =0,
\end{aligned}
$$

on solutions of $\Delta=0$. To simplify this, we use the form (1.9) to rewrite $\Delta$ using the cyclicity and linearity of trace and the properties of the classical adjoint:

$$
\Delta=\frac{1}{m} \frac{1}{\operatorname{det} H} \operatorname{tr}\left(H U H H^{\dagger}\right)
$$

and then, by associativity,

$$
\Delta=\frac{1}{m(m-1)} \frac{1}{\operatorname{det} H} \operatorname{tr}\left(S H^{\dagger}\right)
$$

On comparison with (1.6) we find that $S=m(m-1) \operatorname{det} H G$ and the "on-shell" symmetry condition is satisfied by any $S=($ some scalar factor $) \times \operatorname{det} H G$. This produces a symmetry condition,

$$
\phi_{i} \phi_{j} \frac{\partial^{2} Q}{\partial \phi_{i} \partial \phi_{j}}=0,
$$

which is satisfied by any $Q$ homogeneous of degree zero or one in the first derivatives of $\phi$. This confirms the facts deduced earlier from the Bateman hierarchy.

According to a theorem of Kumei and Bluman [14, the existence of a generalised symmetry which depends on the solution to a linear equation such as (3.13) guarantees the existence of a linearising transform for (1.4). This is indeed the case, as demonstrated in [4]. 
Having disposed of the term with $m$ second derivatives, we need to equate the term with $m-1$ second derivatives to zero. The relevant equation can be written as:

$$
H_{i j}^{\dagger} \frac{\partial Q}{\partial x_{i}} \phi_{j}+2(m-1) U_{k l} \delta_{(i j)(k l)}\left(\frac{\partial^{2} Q}{\partial x_{i} \partial \phi_{n}} \phi_{j n}+\frac{\partial^{2} Q}{\partial \phi \partial \phi_{n}} \phi_{i} \phi_{j n}\right)=0 .
$$

This can be solved by a characteristic of the form,

$$
Q=g(\phi) F(\eta)
$$

where $g$ is an arbitrary smooth, real-valued function, $\eta=x_{i} \phi_{i}$ (no sum) and $F$ is a smooth, real-valued function which respects the homogeneity properties that we have decided on for $Q$. Notice that the diffeomorphism symmetry of the equation is included in this solution.

Finally, to get rid of the remaining terms, we use (3.15) and arrive eventually at the condition:

$$
\varepsilon_{i_{1} \ldots i_{m}} \varepsilon_{j_{i} \ldots j_{m}} \phi_{i_{1}} \phi_{j_{1}} \phi_{i_{2} j_{2}} \ldots \phi_{i} \phi_{j} \ldots \phi_{i_{m} j_{m}}\left(g F^{\prime \prime}+2 g^{\prime} F^{\prime}+g^{\prime \prime} F\right)=0
$$

This is identically true due to the antisymmetry of the $\varepsilon$ symbol.

\section{Conclusion}

The generic Euler hierarchy and the special case of the Bateman hierarchy have been interpreted as a sequence of iterated Lie derivatives for the distinguished symmetries of a large class of Lagrangians. It has been shown that the Bateman hierarchy alone admits explicit dependency on the field due to its homogeneity properties, but the form of the generic hierarchy is unchanged from the analysis in [⿴囗⿰丿丨丁]. The termination of both hierarchies is guaranteed by the generalised symmetries of their associated universal equations, which in turn imply the existence of an infinite class of conservation laws for each equation.

Many questions remain unanswered about the geometrical meaning of the iteration of the Lie derivatives, and the presence of any helpful algebraic properties. It also remains to apply the procedure to more general geometrical constructions than the trivial bundle 
$\pi$ considered here. It is noted that other equations follow from similar procedures, namely certain multidimensional generalisations of the Born-Infeld equation [15].

\section{Acknowledgements}

I am very grateful to D.B. Fairlie for much advice and encouragement. This work was financially supported by the Department of Education for Northern Ireland. 


\section{References}

[1] D.B. Fairlie, J. Govaerts, and A. Morozov. Nucl. Phys. B373 214-232, 1992.

[2] D.B. Fairlie and J. Govaerts. Phys. Lett. 281B 49-53, 1992.

[3] D.B. Fairlie and J. Govaerts. J. Math. Phys. 33 3543-3546, 1992.

[4] D.B. Fairlie and J. Govaerts. J. Phys. A 26 3339-3347, 1993.

[5] J.A. Mulvey. BiHamiltonian formulations of the Bateman equation. To be published in Phys. Lett. A, 1995.

[6] P.J. Olver. Application of Lie Groups to Differential Equations. Springer-Verlag, 2nd edition, 1993.

[7] I.M. Anderson. Contemp. Math. 132 51-73, 1992.

[8] I.M. Anderson. Archivum Mathematicum (Brno) 24 181-202, 1988.

[9] I.M. Anderson and J. Pohjanpelto. Math. Ann. 299 191-222, 1994.

[10] I.M. Anderson and J. Pohjanpelto. Math. Ann. 301 627-653, 1995.

[11] D.R. Grigore. J. Phys. A 28 2921-2937, 1995.

[12] D.R. Grigore. J. Phys. A 28 L49-L57, 1995.

[13] D.J. Saunders. The Geometry of Jet Bundles. Cambridge University Press, 1989.

[14] S. Kumei and G. Bluman. SIAM J. Appl. Math. 42 1157-1173, 1982.

[15] D.B. Fairlie and J.A. Mulvey. J. Phys. A 27 1317-1324, 1994. 